SNUTP 01/034

\title{
Wave function of the radion in the brane background with a massless scalar field and a self-tuning problem
}

\author{
${ }^{(a)}$ Jihn E. Kim*, ${ }^{(b)}$ Bumseok Kyae ${ }^{\dagger}$ and ${ }^{(a)}$ Hyun Min Lee ${ }^{\ddagger}$ \\ ${ }^{(a)}$ Department of Physics and Center for Theoretical Physics, Seoul National University, Seoul \\ 151-747, Korea \\ ${ }^{(b)}$ Bartol Research Institute, University of Delaware, Newark, DE 19716, USA
}

\begin{abstract}
We consider flat solutions in the brane background with a massless scalar field appearing in $5 \mathrm{D} H_{M N P Q}^{2}$. Since there exist bulk singularities or arises the divergent 4D Planck mass, we should introduce a compact extra dimension, the size of which is then fixed by brane tension(s) and a bulk cosmological constant. Inspecting scalar perturbations around the flat solutions, we find that the flat solutions are stable vacua from the positive mass spectrum of radion. We show that the massless radion mode is projected out by the boundary condition arising in cutting off the extra dimension. Thus, the fixed extra dimension is not alterable, which is not useful toward a self-tuning
\end{abstract}

\footnotetext{
*jekim@phyp.snu.ac.kr

†bkyae@bartol.udel.edu

${ }^{\ddagger}$ minlee@phya.snu.ac.kr
} 
of the cosmological constant.

11.25.Mj, 12.10.Dm, 98.80.Cq

Typeset using REVTEX 


\section{INTRODUCTION}

The Randall-Sundrum(RS) models [1,2] have been initially motivated for solving the hierarchy problem in a geometric way [1] and giving an alternative to the conventional Kaluza-Klein compactification of the extra dimension through the normalizable zero mode of graviton [2]. On the other hand, the fact that there exists the 4D flat solution in the RS models even with nonzero brane tension(s) and a nonzero negative bulk cosmological constant reminds us of the cosmological constant problem in a new perspective and thus the RS models deserve further investigations for searching a higher dimensional solution to the cosmological constant problem [3 [12]. The RS solutions requires one (or two) finetuning conditions between brane tension(s) and a bulk cosmological constant by consistency. Therefore, if one loosen the fine-tuning condition(s), our universe should appear as being curved. Therefore, we should seek for models of evading fine-tuning parameters with the requirements for the consistent solution to the cosmological constant problem such that

- there should be no naked singularities and

- the 4D Planck mass should be finite for gravity to be relevant.

Early attempts with a massless bulk scalar field coupled to the brane in the flat bulk ended with the naked singularity and reproduced a fine-tuning in regularizing the naked singularity with another brane [4.5]. The main motivation for introducing a massless scalar field is to use the shift symmetry of the scalar field to self-tune the cosmological constant with its special coupling to the brane.

On the other hand, there have appeared suggestions of introducing a massless bulk scalar field not coupled to the brane [8,10,11]. In those attempts, the scalar field just plays a role of bulk source only and thus it gives rise to only a non-multiplicative integration constant of the warp factor by making bulk solutions non-trivial. [The integration constant from scalar field itself is not important since it is just contained in the warp factor as multiplicative.] With $1 / H^{2}$ term, where $H^{2}=H_{M N P Q} H^{M N P Q}$, it has been shown that there exists a self-tuning solution [9]. 
However, attempts with a conventional kinetic term of the scalar field did not give a selftuning solution, i.e. there appear naked singularities or the warp factor becomes divergent away from the brane. Therefore, it is necessary to introduce another brane for cutting off the extra dimension, from which we concluded that there does not exists a self-tuning solution when the scalar couples to the brane [5]. However, when the scalar does not couple to the brane, another brane introduces a parameter the distance between two branes, as a result of which it seems that there does not arise any direct fine-tunings but the extra dimensional distance is determined by brane tensions and bulk cosmological constant. In this case, there arises a question whether the size of the extra dimension can be regarded as a kind of integration constant to be determined by the boundary condition.

In this paper, we study this question whether the extra dimensional distance can be regarded as an integration constant or not by investigating scalar perturbations around the flat solutions obtained for arbitrary bulk cosmological constant. We find that the mass spectrum of the radion is given as positive definite and a massless mode is decoupled by the boundary condition appearing in cutting off the extra dimension. Thus the flat solutions are shown to be true minima of the action in taking into account the boundary condition.

The absence of massless radion means that the size of extra dimension is fixed but not alterable. Therefore, since the size of extra dimension does not correspond to a kind of integration constant in the self-tuning models, one cannot evade one fine-tuning condition between brane tension(s) and bulk cosmological constant for obtaining a flat solution with a conventional kinetic energy term.

Using the remaining gauge transformations preserving the scalar perturbations, we also find that there exist a massless graviscalar and a massless spin-2 graviton while there is no massless vector mode. The massive excitations of spin-2 graviton are shown to be positive, which guarantees the stability of the flat solutions again.

This paper is organized as follows. In the next section, we provide the model setup for consideration and present flat solutions. In Sec. III, we perturb the scalar field around the flat solutions and identify the radion spectrum. Then, in Sec. IV, we present the graviton 
perturbations for completeness. Sec. V is a conclusion.

\section{MODEL SETUP}

On top of the RS model [1,2], we introduce a three form field in the bulk without coupling to the brane. For future convenience of cutting off the extra dimension, we include the sum of brane actions. Then, the 5D action of our model setup is

$$
S=\int d^{4} x \int d y \sqrt{-g}\left(\frac{M^{3}}{2} R-\Lambda_{b}-\frac{1}{2 \cdot 4 !} H_{M N P Q} H^{M N P Q}+\sum_{i} \mathcal{L}_{m}^{(i)} \delta\left(y-y_{i}\right)\right) .
$$

In order to get $4 \mathrm{D}$ flat solutions, let us take the ansatz for the metric as

$$
d s^{2}=\beta^{2}(y) \eta_{\mu \nu} d x^{\mu} d x^{\nu}+d y^{2}
$$

where $\left(\eta_{\mu \nu}\right)=$ diag. $(-1,+1,+1,+1)$. Then Einstein tensors are,

$$
\begin{aligned}
& G_{\mu \nu}=g_{\mu \nu}\left[3\left(\frac{\beta^{\prime}}{\beta}\right)^{2}+3\left(\frac{\beta^{\prime \prime}}{\beta}\right)\right], \\
& G_{55}=6\left(\frac{\beta^{\prime}}{\beta}\right)^{2} .
\end{aligned}
$$

where prime denotes differentiation with respect to $y$. With the brane tension $\Lambda_{1}$ and $\Lambda_{2}$ at the $y=0$ and $y=y_{c}$ branes, respectively, and the bulk cosmological constant $\Lambda_{b}$, the energy momentum tensors are

$$
\begin{aligned}
T_{M N} & =-g_{M N} \Lambda_{b}-\frac{\sqrt{-g^{(4)}}}{\sqrt{-g}} g_{\mu \nu} \delta_{M}^{\mu} \delta_{N}^{\nu} \sum_{i} \Lambda_{i} \delta\left(y-y_{i}\right)+\tilde{T}_{M N}, \\
\tilde{T}_{M N} & =\frac{1}{4 !}\left(4 H_{M P Q R} H_{N} P Q R-\frac{1}{2} H^{2} g_{M N}\right) \\
& =\nabla_{M} \phi \nabla_{N} \phi-\frac{1}{2} g_{M N}(\nabla \phi)^{2},
\end{aligned}
$$

where we used the fact that a three form field in 5D spacetime is dual to a scalar field as $H_{M N P Q}=\sqrt{-g} \epsilon_{M N P Q}{ }^{R} \nabla_{R} \phi$. Here when the dual relation is inserted in the kinetic term of the action, its overall sign looks opposite compared with the case of a scalar field but there

does not arise such inconsistency between the action and the Einstein's equation when the surface term for the three form field is included. 
We also take the ansatz for the nonvanishing components of four form field $H_{\mu \nu \rho \sigma}$ as

$$
H_{\mu \nu \rho \sigma}=\sqrt{-g} \epsilon_{\mu \nu \rho \sigma} f(y)
$$

where $\mu, \cdots$ run over the Minkowski indices $0,1,2$, and 3 . With the above ansatz, the field equation for the four form field is satisfied,

$$
\partial_{M}\left[\sqrt{-g} H^{M N P Q}\right]=0
$$

The two relevant Einstein equations are the $(55)$ and $(\mu \mu)$ components,

$$
\begin{aligned}
6\left(\frac{\beta^{\prime}}{\beta}\right)^{2} & =-\Lambda_{b}+\frac{A}{\beta^{8}} \\
3\left(\frac{\beta^{\prime}}{\beta}\right)^{2}+3\left(\frac{\beta^{\prime \prime}}{\beta}\right) & =-\Lambda_{b}-\Lambda_{1} \delta(y)-\Lambda_{2} \delta\left(y-y_{c}\right)-\frac{A}{\beta^{8}}
\end{aligned}
$$

where $A / \beta^{8} \equiv f^{2} / 2$ expressed in terms of a 'positive' constant $A$. The solutions of Eq. (9) and (10) with $Z_{2}$ symmetry are [10]

$$
\begin{aligned}
\text { for } \quad \Lambda_{b}<0 \quad \text { (1) } \beta(|y|) & =\left(\frac{a}{k}\right)^{1 / 4}[\sinh (-4 k|y|+c)]^{1 / 4} \\
(2) \beta(|y|) & =\left(\frac{a}{k}\right)^{1 / 4}[\sinh (4 k|y|+c)]^{1 / 4} \\
\text { for } \quad \Lambda_{b}>0 \quad: \quad(1) \beta(|y|) & =\left(\frac{a}{k}\right)^{1 / 4}[\sin (-4 k|y|+c)]^{1 / 4} \\
\text { (2) } \beta(|y|) & =\left(\frac{a}{k}\right)^{1 / 4}[\cos (-4 k|y|+c)]^{1 / 4} \\
\text { for } \quad \Lambda_{b}=0 \quad: \quad \text { (1) } \beta(|y|) & =(-4 a|y|+c)^{1 / 4} \\
\text { (2) } \beta(|y|) & =(4 a|y|+c)^{1 / 4}
\end{aligned}
$$

where $k \equiv \sqrt{\left|\Lambda_{b}\right| / 6}$ and the $a$ is defined in terms of $A$,

$$
a \equiv \sqrt{\frac{A}{6}} .
$$

We note that $\beta$ 's of Eqs. (12) and (16) do not give localized gravity on the $y=0$ brane while $\beta$ 's of Eqs. (11), (13), (15) have naked singularities at $|y|=c /(4 k)$ or $|y|=c /(4 a)$ and $\beta$ of Eq. (14) does at $|y|=(c+\pi / 2) /(4 k)$. Therefore, to get the effective four dimensional gravity or to avoid the sigularities in the bulk, it is indispensable to cut the extra dimension such that it has a finite length size by introducing another brane. 
Then, since the $Z_{2}$ symmetry and the periodicity for the compact dimension gives rise to the boundary conditions at the branes,

$$
\left.\frac{\beta^{\prime}}{\beta}\right|_{y=y_{i}^{+}} \equiv-\frac{\Lambda_{i}}{6},
$$

the consistency requires the following relations for the above three cases

$$
\begin{aligned}
& \text { for } \Lambda_{b}<0: \quad \pm c=\operatorname{coth}^{-1}\left(\frac{k_{1}}{k}\right)=4 k y_{c}-\operatorname{coth}^{-1}\left(\frac{k_{2}}{k}\right) \\
& \text { for } \quad \Lambda_{b}>0: \quad(1) c=\cot ^{-1}\left(\frac{k_{1}}{k}\right)=4 k y_{c}-\cot ^{-1}\left(\frac{k_{2}}{k}\right) \\
& \qquad(2) c=-\tan ^{-1}\left(\frac{k_{1}}{k}\right)=4 k y_{c}+\tan ^{-1}\left(\frac{k_{2}}{k}\right) \\
& \text { for } \quad \Lambda_{b}=0: \quad \pm c=\frac{a}{k_{1}}=a\left(4 y_{c}-\frac{1}{k_{2}}\right),
\end{aligned}
$$

where $c>0$ for $\Lambda_{b} \leq 0$ and $0<c<\pi / 2$ for $\Lambda_{b}>0$ and

$$
k_{1} \equiv \frac{\Lambda_{1}}{6}, k_{2} \equiv \frac{\Lambda_{2}}{6}
$$

For all the above cases, there do not appear any direct fine-tuning conditions between brane tensions unlike the RS case but the size of the extra dimension is determined by the brane tensions and the bulk cosmological constant. In particular, for the $\Lambda_{b}>0$ case (Eq. (14)), as recently argued in Ref. [11], it may be possible to compactify the extra dimension without the need of introducing another brane by identifying the two extrema of the warp factor symmetric around the $y=0$ brane. In Fig. 1 we show a schematic behavior of the warp factor. If Point Q corresponds to the location of the another brane, then one has to introduce a brane tension there. However, if Point $\mathrm{P}$ is chosen, it is not necessary to introduce another brane. That means the extra dimensional size is up to Point $\mathrm{P}$ which is determined by the other parameters in the theory. For this to be the case, we must have a shift symmetry in the radion direction whose distance is determined by the parameters in the theory. Namely, we only have to take the boundary condition as the first one in Eq. (21) and the length size of the extra dimension can be regarded as being determined by the brane tension as $y_{c}=c /(4 k)=-(4 k)^{-1} \tan ^{-1}\left(k_{1} / k\right)$ for $k_{1}<0$. 
FIGURES

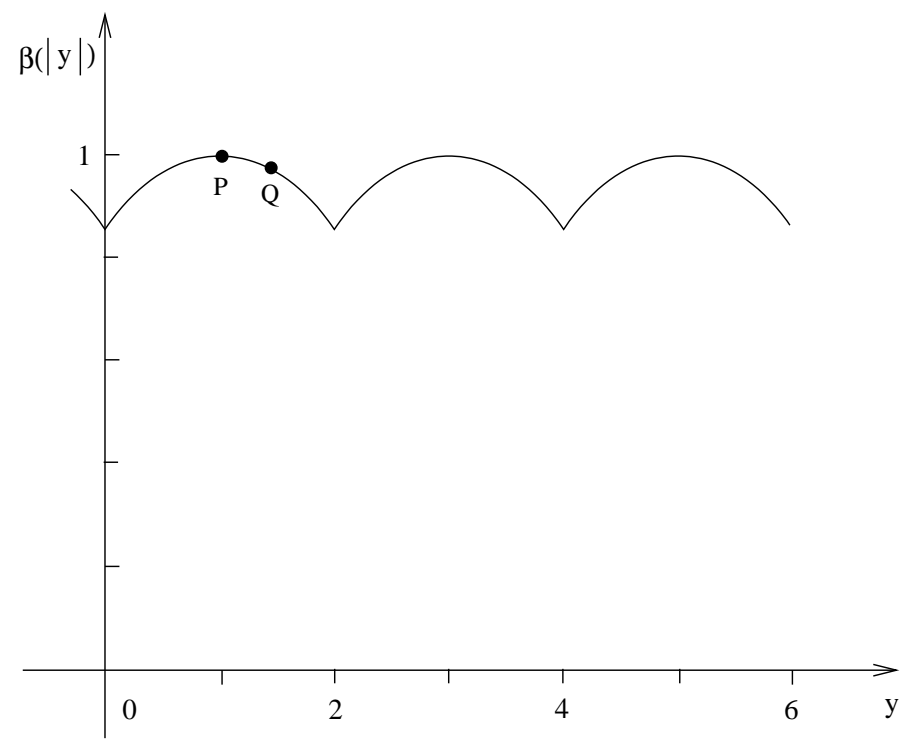

Fig. 1. The schematic behavior of the warp factor for $\Lambda_{b}>0$. Point $P$ is the case of our main concern.

\section{SCALAR PERTURBATIONS AND RADION}

We expect that there exists the radion mode as a perturbation around the $4 \mathrm{D}$ flat solutions obtained, which corresponds to altering the positions of the branes or the size of the extra dimension and appears a scalar field in the effective 4D theory. Thus let us make scalar perturbations around the flat solutions for investigating the radion spectrum in our model.

In the RS case without radius stabilization, there exists a massless radion for the flat solution since the size of extra dimension is not determined [1,13,14]. And it is also shown that the radion has a positive mass for the $4 \mathrm{D}$ AdS solution while it has a negative mass for the $4 \mathrm{D}$ dS solution [15,16]. For the stabilized RS model, the radion mass is also investigated in detail in Ref. [17]. In models with metastable graviton and multigravity scenarios, it is shown that the radion dynamics is crucial to test their stability [18, [19].

As we mentioned in the previous section, we regard the three form field as a scalar field 
by duality on investigating perturbations for convenience. In that case, we only have to deal with the energy momentum tensor coming from the scalar field as in Eq. (6) and the scalar field equation such as

$$
\partial_{M}\left(\sqrt{-g} \partial^{M} \phi\right)=0 .
$$

We thus take a general ansatz for the metric perturbations as

$$
\begin{aligned}
d s^{2} & =K^{-2}(z)\left(\eta_{M N}+h_{M N}(x, z)\right) d x^{M} d x^{N} \\
\phi(x, z) & =\phi_{0}(z)+\varphi(x, z),
\end{aligned}
$$

where $z=\int^{z} d y / \beta(y), K(z)=1 / \beta(y(z))$ and $\left(\phi_{0}^{\prime}\right)^{2}=A K^{6}$.

Then, the linearized Einstein's tensor, the linearized energy momentum tensor and the linearized scalar equation read

$$
\begin{aligned}
& \delta G_{M N}=-\frac{\square}{2} \bar{h}_{M N}+\partial_{(M} \partial^{P} \bar{h}_{N) P}-\frac{1}{2} \eta_{M N} \partial^{P} \partial^{Q} \bar{h}_{P Q}-\frac{3 K^{\prime}}{2 K}\left(\partial_{M} h_{N 5}+\partial_{N} h_{M 5}-\partial_{5} h_{M N}\right) \\
&-3 \eta_{M N}\left[\left(-\frac{K^{\prime \prime}}{K}+2 \frac{K^{\prime 2}}{K^{2}}\right) h_{55}-\frac{K^{\prime}}{K} \partial^{P} \bar{h}_{P 5}\right]-3\left[\frac{K^{\prime \prime}}{K}-2 \frac{K^{\prime 2}}{K^{2}}\right] h_{M N} \\
& \delta T_{\mu \nu}=\left(-3 \frac{K^{\prime \prime}}{K}+6 \frac{K^{\prime 2}}{K^{2}}\right) h_{\mu \nu}+\sum_{i} \delta\left(z-z_{i}\right) \frac{\Lambda_{i}}{2 K} h_{55} \eta_{\mu \nu}+\frac{1}{2}\left(\phi_{0}^{\prime}\right)^{2} h_{55} \eta_{\mu \nu}-\phi_{0}^{\prime} \varphi^{\prime} \eta_{\mu \nu} \\
& \delta T_{\mu 5}= 6 \frac{K^{\prime 2}}{K^{2}} h_{\mu 5}+\phi_{0}^{\prime} \partial_{\mu} \varphi \\
& \delta T_{55}= 6 \frac{K^{\prime 2}}{K^{2}} h_{55}-\frac{1}{2}\left(\phi_{0}^{\prime}\right)^{2} h_{55}+\phi_{0}^{\prime} \varphi^{\prime}, \\
& \square \varphi-3 \frac{K^{\prime}}{K} \varphi^{\prime}+\frac{1}{2} \phi_{0}^{\prime}\left(h_{\mu}^{\mu \prime}-h_{55}^{\prime}\right)=0
\end{aligned}
$$

where $(M, N)$ is a half of the symmetric combination, ' denotes the derivative with respect to $z$ and $\partial^{M} \equiv \eta^{M N} \partial_{N}, \square \equiv \eta^{\mu \nu} \partial_{\mu} \partial_{\nu}+\partial_{z}^{2} . \bar{h}_{M N}$ is defined as $\bar{h}_{M N} \equiv h_{M N}-\eta_{M N} h / 2$ and $h$ is the trace for $h_{M N}$.

Since we are interested in the scalar perturbations for investigating the radion, let us take a gauge choice in the metric ansatz (25) as

$$
h_{\mu \nu}(x, z)=F(x, z) \eta_{\mu \nu}, h_{\mu 5}=0, h_{55}(x, z)=G(x, z)
$$


Thus, the $(\mu \nu)$ component of the linearized Einstein tensor is written as the form

$$
\delta G_{\mu \nu}=\partial_{\mu} \partial_{\nu}\left(-F-\frac{1}{2} G\right)+\cdots
$$

where the ellipses all contain terms proportional to $\eta_{\mu \nu}$. And the linear perturbations from the energy momentum tensor are also $\sim \eta_{\mu \nu}$. Therefore, we obtain an immediate relation such as

$$
G=-2 F
$$

Then the linearized $(\mu \mu),(\mu 5)$ and (55) Einstein's equations and the linearized scalar field equation are

$$
\begin{gathered}
\frac{3}{2} F^{\prime \prime}-\frac{15}{2} \frac{K^{\prime}}{K} F^{\prime}+6\left[2\left(\frac{K^{\prime}}{K}\right)^{2}-\frac{K^{\prime \prime}}{K}\right] F+\left[\sum_{i} \frac{\Lambda_{i}}{K} \delta\left(z-z_{i}\right)+\left(\phi_{0}^{\prime}\right)^{2}\right] F+\phi_{0}^{\prime} \varphi^{\prime}=0 \\
-\frac{3}{2} \partial_{\mu} F^{\prime}-3 \frac{K^{\prime}}{K} \partial_{\mu} F=\phi_{0}^{\prime} \partial_{\mu} \varphi \\
\frac{3}{2} \partial^{\mu} \partial_{\mu} F-6 \frac{K^{\prime}}{K} F^{\prime}+\left[12\left(\frac{K^{\prime}}{K}\right)^{2}-\left(\phi_{0}^{\prime}\right)^{2}\right] F-\phi_{0}^{\prime} \varphi^{\prime}=0 \\
\partial^{\mu} \partial_{\mu} \varphi+\varphi^{\prime \prime}-3 \frac{K^{\prime}}{K} \varphi^{\prime}+3 \phi_{0}^{\prime} F^{\prime}=0 .
\end{gathered}
$$

The $(\mu 5)$ component equation (36) can be integrated to give

$$
\phi_{0}^{\prime} \varphi=-\frac{3}{2}\left(F^{\prime}-2 \frac{K^{\prime}}{K} F\right)+f(z) .
$$

The metric ansatz (32), Eqs. (34) and (39) with $f(z)=0$ fix our gauge choice. Eliminating $\phi_{0}^{\prime} \varphi^{\prime}$ from Eqs. (35) and (37) gives rise to the equation for $F$ only as

$$
\partial^{\mu} \partial_{\mu} F+F^{\prime \prime}-9 \frac{K^{\prime}}{K} F^{\prime}+\left[16\left(\frac{K^{\prime}}{K}\right)^{2}-4 \frac{K^{\prime \prime}}{K}+\sum_{i} \frac{2 \Lambda_{i}}{3 K} \delta\left(z-z_{i}\right)\right] F=0
$$

from which we can easily obtain the boundary conditions at the branes

$$
\left[F^{\prime}-2 \frac{K^{\prime}}{K} F\right]_{z=z_{i}^{+}}=0,
$$


where we used $K^{\prime \prime} / K=2 A K^{6} / 3+\left(\Lambda_{i} / 3 K\right) \delta\left(z-z_{i}\right)$ and $\Lambda_{i}=\left.6 K^{\prime}\right|_{z=z_{i}}$. It is staightforward to check the scalar field equation (38) by multiplying Eq. (38) with $\phi_{0}^{\prime}$ and using Eqs. (39), (37) and the background equations (9), (10) in the $z$ coordinate. Thus, $F$ must satisfy Eq. (41) and the bulk equation

$$
\partial^{\mu} \partial_{\mu} F+F^{\prime \prime}-9 \frac{K^{\prime}}{K} F^{\prime}+\left[16\left(\frac{K^{\prime}}{K}\right)^{2}-\frac{4 K^{\prime \prime}}{K}\right] F=0
$$

Note that studying $F$ is equivalent to studying the radion, in view of Eqs. (32) and (34).

To make a Kaluza-Klein reduction of the radion field to 4D, we choose a separation of variables as $F(x, z)=\psi(z) \rho(x)$. Taking a rescaling such as $\tilde{\psi}=\psi /\left(\phi_{0}^{\prime} K^{3 / 2}\right)$ [20], we obtain the $4 \mathrm{D}$ equation of the radion field and the bulk equation for the wave function of the radion

$$
\begin{aligned}
\left(\partial^{\mu} \partial_{\mu}-m^{2}\right) \rho(x) & =0, \\
\left(-\partial_{z}^{2}+V(z)\right) \tilde{\psi}(z) & =m^{2} \tilde{\psi}(z)
\end{aligned}
$$

where

$$
\begin{aligned}
V(z) & =\xi\left(\frac{1}{\xi}\right)^{\prime \prime}, \\
\xi & =\frac{\phi_{0}^{\prime}}{K^{1 / 2} K^{\prime}} .
\end{aligned}
$$

The above equation corresponds to nothing but a supersymmetric quantum mechanics

$$
Q^{\dagger} Q \tilde{\psi}(z) \equiv\left[\partial_{z}+\xi\left(\frac{1}{\xi}\right)^{\prime}\right]\left[-\partial_{z}+\xi\left(\frac{1}{\xi}\right)^{\prime}\right] \tilde{\psi}(z)=m^{2} \tilde{\psi}(z)
$$

The hermitianity of the above differential operator guarantees the positivity of the mass spectrum with $m^{2} \geq 0$ : there is no tachyonic mode of radion. The bulk solution for the massless radion field with $m^{2}=0$ is given by a linear combination such as

$$
\tilde{\psi}_{0}(z)=\frac{1}{\xi}\left(c_{0}+d_{0} \int_{0}^{z} d z \xi^{2}\right)
$$

or

$$
\psi_{0}(z)=K^{2} K^{\prime}\left[c_{0}+d_{0} \int_{0}^{z} d z\left(\frac{\phi_{0}^{\prime}}{K^{1 / 2} K^{\prime}}\right)^{2}\right]
$$


where $c_{0}$ and $d_{0}$ are integration constants. Then, we can take the wave function of the massless radion to be consistent with the $Z_{2}$ symmetry

$$
\psi_{0}(z)=\left\{\begin{array}{l}
K^{2} K^{\prime}\left[c_{0}+d_{0} \int_{0}^{z} d z\left(\frac{\phi_{0}^{\prime}}{K^{1 / 2} K^{\prime}}\right)^{2}\right] \text { for } z>0, \\
-K^{2} K^{\prime}\left[c_{0}-d_{0} \int_{0}^{z} d z\left(\frac{\phi_{0}^{\prime}}{K^{1 / 2} K^{\prime}}\right)^{2}\right] \text { for } z<0 .
\end{array}\right.
$$

Then, using the boundary condition at the $z=0$ (or $y=0$ ) brane from Eq. (41),

$$
\left[\psi^{\prime}-2 \frac{K^{\prime}}{K} \psi\right]_{z=0^{+}}=0
$$

we obtain the ratio between two integration constants as

$$
\text { (A) : } \frac{c_{0}}{d_{0}}=-\left[\frac{\left(\phi_{0}^{\prime}\right)^{2}}{K^{\prime \prime} K^{\prime} K}\right]_{z=0^{+}} \text {. }
$$

On the other hand, since the extra dimension should be cut off with or without another brane to escape a bulk singularity or a divergent 4D Planck mass, an additional boundary condition appears. For the case without another brane, the derivative of the metric perturbation should be zero at the end of the extra dimension as for the background metric. Therefore, in view of Eq. (41), another boundary condition should be the same irrespective of the existence of an additional brane as

$$
\left.\left(K^{-2} \psi\right)^{\prime}\right|_{z=z_{c}^{-}}=0
$$

Thus we also find another necessary condition for integration constants

$$
\text { (B) }: \frac{c_{0}}{d_{0}}=-\left[\frac{\left(\phi_{0}^{\prime}\right)^{2}}{K^{\prime \prime} K^{\prime} K}\right]_{z=z_{c}^{-}}-\int_{0}^{z_{c}} d z\left(\frac{\phi_{0}^{\prime}}{K^{1 / 2} K^{\prime}}\right)^{2} \text {. }
$$

However, the two equations (52) and (54) cannot be satisfied simultaneously because the difference $(A)-(B)$ between the two turns out to be nonzero

$$
(A)-(B)=-3 \int_{0}^{z_{c}} d z K^{-3} \neq 0 .
$$

As a result, the massless radion mode should be regarded as being projected out by another boundary condition appearing in cutting off the extra dimension, irrespective of whether the extra dimension is compactified with one or two branes. 
The absence of massless radion implies that it is impossible to change the brane spacing or the size of extra dimension for the flat solution to exist for different sets of brane tension(s) and bulk cosmological constant. Thus the size of extra dimension does not correspond to an integration constant in the self-tuning models and one cannot escape the one fine-tuning condition [10] between input parameters for obtaining a flat solution for initially fixed extra dimension as seen in Eqs. (19)-(22).

On the other hand, massive radion modes are alive with discrete positive masses since there appear generically two integration constants and mass to be determined : one integration constant and mass are determined by two boundary conditions at the ends of the extra dimension and the other integration constant is fixed by normalization of the wave function of the radion. Therefore, the size of the extra dimension fixed by Eqs. (19)-(22) is stable under perturbations, which guarantees that the flat solutions of the Einstein's equations Eqs. (11)-(16) are true minima of the action.

\section{GRAVITON PERTURBATIONS}

Even though we made a gauge choice such that only scalar perturbations are taken into account in the previous section, there should be the remaining gauge transformations preserving the gauge conditions (34) and (39) with the metric ansatz (32), which enables us to impose a convenient 4D gauge for the graviton. Thus, for completeness, on top of the scalar perturbations corresponding to the radion in the previous section, let us consider the graviton perturbations with the general metric ansatz

$$
h_{\mu \nu}(x, z)=F(x, z) \eta_{\mu \nu}+\tilde{h}_{\mu \nu}(x, z), h_{\mu 5} \neq 0, h_{55}(x, z)=G(x, z) .
$$

Then, with the gauge conditions (34) and (39) for the scalar perturbations, we use a 4D gauge transformations to impose the de Donder gauge condition for the graviton

$$
0=\partial^{\mu} \overline{\tilde{h}}_{\mu \nu}=\partial^{\mu} \bar{h}_{\mu \nu}
$$


where $\bar{h}_{\mu \nu} \equiv h_{\mu \nu}-\frac{1}{2} h_{\lambda}^{\lambda} \eta_{\mu \nu}$ and the second equality comes from Eq. (34). Now we have fixed five of the fifteen degrees of freedom of the 5D metric perturbations.

Assuming that the equations for scalar perturbations (35)-(38) are reproduced for the general gauge choice with Eqs. (56) and (57), we obtain the equations for the graviton from (27)-(30) as

$$
\begin{gathered}
-\frac{1}{2}\left(\square-3 \frac{K^{\prime}}{K^{\prime}} \partial_{5}\right) \tilde{h}_{\mu \nu}+\frac{1}{4} \eta_{\mu \nu}\left(\square+\partial_{5}^{2}-6 \frac{K^{\prime}}{K} \partial_{5}\right) \tilde{h}_{\mu}^{\mu} \\
+\left(\partial_{5}-3 \frac{K^{\prime}}{K}\right)\left(\partial_{(\mu} h_{\nu) 5}-\eta_{\mu \nu} \partial^{\lambda} h_{\lambda 5}\right)=0, \\
-\frac{1}{2}\left(\partial^{\lambda} \partial_{\lambda}+6 \frac{K^{\prime \prime}}{K}\right) h_{\mu 5}+\frac{1}{2} \partial_{\mu}\left(\partial^{\lambda} h_{5 \lambda}-\frac{1}{2} \partial_{5} \tilde{h}_{\lambda}^{\lambda}\right)=0, \\
\frac{1}{4} \partial^{\lambda} \partial_{\lambda} \tilde{h}_{\mu}^{\mu}+\frac{3 K^{\prime}}{K}\left(\partial^{\lambda} h_{\lambda 5}-\frac{1}{2} \partial_{5} \tilde{h}_{\mu}^{\mu}\right)=0 .
\end{gathered}
$$

Then we find that both the trace of Eq. (58) and Eq. (60) can be satisfied simultaneously with

$$
\partial^{\lambda} h_{5 \lambda}-\frac{1}{2} \partial_{5} \tilde{h}_{\mu}^{\mu}=0
$$

under which Eqs. (60) and (59) become, respectively,

$$
\begin{gathered}
\partial^{\lambda} \partial_{\lambda} \tilde{h}_{\mu}^{\mu}=0, \\
\left(\partial^{\lambda} \partial_{\lambda}+6 \frac{K^{\prime \prime}}{K}\right) h_{\mu 5}=0 .
\end{gathered}
$$

Therefore, there appears a massless gravi-scalar field $\tilde{h}_{\mu}^{\mu}$, which couples to the trace of the 4D energy momentum tensor as for the radion. On the other hand, since Eq. (63) cannot be satisfied either on the brane or in the bulk, $h_{\mu 5}=0$, i.e., there is no massless vector mode, which is consistent with Eq. (61) since $\left(\tilde{h}_{\mu}^{\mu}\right)^{\prime}=0$ from comparing the linearized scalar equations (31) and (38).

¿From Eq. (58), we also observe that the transverse traceless spin-2 graviton $\left(\tilde{h}_{\mu \nu}^{T T}\right)$ is automatically decoupled due to $\tilde{h}_{\mu}^{\mu \prime}=h_{\mu 5}=0$ and the de Donder gauge (57): 


$$
\left(\square-3 \frac{K^{\prime}}{K} \partial_{5}\right) \tilde{h}_{\mu \nu}^{T T}=0
$$

Consequently, it turns out that there exists a massless spin-2 graviton since the above equation is satisfied by $\tilde{h}_{\mu \nu}^{T T}(x, z)=c_{0} e^{i p x} \epsilon_{\mu \nu}$ with $p^{2}=0$, where $c_{0}$ is a constant and $\epsilon_{\mu \nu}$ is a polarization tensor.

With a separation of variables as $\tilde{h}_{\mu \nu}^{T T}=K^{3 / 2}(z) \tilde{\psi}(z) e^{i p x} \epsilon_{\mu \nu}\left(p^{2}=-m^{2}\right)$, we obtain a Schrödinger-like equation again

$$
\left(-\partial_{z}^{2}+V(z)\right) \tilde{\psi}(z)=m^{2} \tilde{\psi}(z)
$$

where

$$
V(z)=\frac{15}{4}\left(\frac{K^{\prime}}{K}\right)^{2}-\frac{3 K^{\prime \prime}}{2 K}
$$

Therefore, we find that $m^{2} \geq 0$, i.e., there is no tachyonic state of the graviton since the above equation can be regarded as a supersymmetric quantum mechanics

$$
Q^{\dagger} Q \tilde{\psi}(z) \equiv\left(\partial_{z}-\frac{3}{2} \frac{K^{\prime}}{K}\right)\left(-\partial_{z}-\frac{3}{2} \frac{K^{\prime}}{K}\right) \tilde{\psi}(z)=m^{2} \tilde{\psi}(z)
$$

\section{CONCLUSION}

In this paper, by introducing a 5D massless scalar field not coupled to the brane, we found that there exist flat solutions without direct fine-tunings between brane tension(s) and a bulk cosmological constant. However, since there arises a naked singularity or the 4D Planck mass becomes divergent for non-compact extra dimension, the extra dimension should be compactified with or without another brane and then the size of extra dimension is fixed by brane and bulk cosmological constants.

At first sight, the size of extra dimension looks a kind of integration constant for the existence of flat solutions without fine-tunings. However, on inspecting scalar perturbations around the flat solutions, the absence of massless radion mode implies that it is impossible 
to use the brane spacing as an integration constant and the self-tuning of the cosmological constant is not realized with the radion.

For completeness, using the remaining gauge transformations preserving the scalar perturbations, we find that there exist a massless gravi-scalar and a massless spin-2 graviton while there is no massless vector mode. The spectrum of massive spin-2 graviton is shown to be non-negative, which implies the stability of the flat solutions with a massless bulk scalar field together with the positivity of the radion spectrum.

\section{ACKNOWLEDGMENTS}

This work is supported in part by the BK21 program of Ministry of Education(JEK, HML), the KOSEF Sundo Grant(JEK, HML), and by the Center for High Energy Physics(CHEP), Kyungpook National University(JEK, HML), and by US Department of Energy DE-FG02-91ER40626(BK). 


\section{REFERENCES}

[1] L. Randall and R. Sundrum, Phys. Rev. Lett. 83, 3370 (1999) hep-th/9905221.

[2] L. Randall and R. Sundrum, Phys. Rev. Lett. 83, 4690 (1999) hep-th/9906064.

[3] V. A. Rubakov and M. E. Shaposhinikov, Phys. Lett. B125, 139 (1983).

[4] N. Arkani-Hamed, S. Dimopoulos, N. Kaloper, and R. Sundrum, Phys. Lett. B480, 193 (2000) hep-th/0001197; S. Kachru, M. Schulz and E. Silverstein, Phys. Rev. D62, 045021 (2000) hep-th/0001206; C. Csaki, J. Erlich, C. Grojean and T. Hollowood, Nucl. Phys. B584, 359 (2000) hep-th/0004133.

[5] S. Forste, Z. Lalak, S. Lavignac and H. P. Nilles, Phys. Lett. B481, 360 (2000) hepth/0002164 and JHEP 0009, 034 (2000) hep-th/0006139.

[6] S.-H. H. Tye and I. Wasserman, Phys. Rev. Lett. 86, 1682 (2001) hep-th/0006068; E. Flanagan, N. Jones, H. Stoica, S.-H. H. Tye, and I. Wasserman, Phys. Rev. D64, 045007 (2001) hep-th/0012129.

[7] A. Kehagias and K. Tamvakis, hep-th/0011006.

[8] H. Collins and B. Holdom, Phys. Rev. D63, 084020 (2001), hep-th/0009127.

[9] J. E. Kim, B. Kyae and H. M. Lee, Phys. Rev. Lett. 86, 4223 (2001) hep-th/0011118.

[10] J. E. Kim, B. Kyae and H. M. Lee, Nucl. Phys. B613, 306 (2001) hep-th/0101027.

[11] S. Hayakawa, T. Hirayama and R. Kitano, hep-th/0108109.

[12] E. E. Flanagan, S.-H. H. Tye and I. Wasserman, hep-th/0110070.

[13] C. Csaki, M. Graesser, L. Randall and J. Terning, Phys. Rev. D62, 045015 (2000) hep-ph/9911406.

[14] C. Charmousis, R. Gregory and V. A. Rubakov, Phys. Rev. D62, 067505 (2000) hepth/9912160. 
[15] Z. Chacko and P. J. Fox, Phys. Rev. D64, 024015 (2001) hep-th/0102023].

[16] P. Binetruy, C. Deffayet and P. Langlois, hep-th/0101234.

[17] C. Csaki, M. L. Graesser and G. D. Kribs, Phys. Rev. D63, 065002 (2001) hepth/0008151].

[18] L. Pilo, R. Rattazzi and A. Zaffaroni, JHEP 0007, 056 (2000) hep-th/0004028.

[19] I. I. Kogan, S. Mouslopoulos, A. Papazoglou and L. Pilo, hep-th/0105255.

[20] M. Giovannini, hep-th/0106041. 